\title{
EDITORIAL
}

\section{Journal of Human Hypertension: Millennium issue}

\section{Introduction/Preface}

This combined issue of the Journal of Human Hypertension consists of a series of detailed review articles by distinguished authors reflecting our current understanding of clinical aspects of hypertension, whilst also looking to the future. It is to be hoped that many researchers, particularly those who are new to the field, will find them useful.

The recent publication of the INSIGHT and NORDIL studies, together with STOP-H2 and CAPP, represent the first of a great many long-term outcome studies comparing different classes of antihypertensive drugs. The first few years of the 21st century will be marked by several similar studies including ALLHAT and ASCOT which will address the question of which are the best antihypertensive drugs for different types of patients. The days of the placebo-controlled trials are almost over. The question now is does it matter how we reduce blood pressure rather than who should we treat. Particular interest will be focused on patients with non-insulin dependent diabetes mellitus (NIDDM) where we still have a lot to learn.

One could argue that in the topic of NIDDM, the world has been turned upside down. It is now best to consider NIDDM purely as a cardiovascular risk factor than an endocrinological condition. Diabetologists should have more in common with lipidiologists and hypertensionologists, or for that matter antismokingologists, rather than with endocrinologists. The UKPDS investigators have taught us a lot.
The salt controversy still bubbles along but the presentation of the impressive results of the DASHsodium study at the American Society of Hypertension meeting in New York this year hopefully will silence the salt nihilists; or perhaps it won't! Similarly, the presentation of the INTERMAP epidemiological study at the International Society of Hypertension meeting in Chicago has shed a great deal more light on nutritional factors causing the wide differences in blood pressure both between and within populations. It's been a busy year for conferences, what with European Society of Hypertension as well!

Turning to the newer antihypertensive agents, it is fair to say that the angiotensin II receptor antagonists have been a spectacular success with their almost complete lack of side-effects at least in the short-term. The long-term outcome studies of this class on cardiovascular events are awaited with great interest. The first will probably be the LIFE trial comparing losartan with atenolol in hypertensive patients with left ventricular hypertrophy. Results should become available in about 2 years time. Meanwhile we have the prospect of yet another new class, the vasopeptidase inhibitors including omapatrilat. Of course they will require long-term outcome studies too, so that should keep us busy until the year 2010. And then what? We live in interesting times.

DG Beevers University Department of Medicine City Hospital, Birmingham, UK 